\title{
Embedding of signed regular graphs
}

\author{
Deepa Sinha and Anita Kumari Rao \\ Department of Mathematics, South Asian University \\ New Delhi-110021, India \\ e-mails: deepa_sinha2001@yahoo.com, \\ anita.rao31@gmail.com
}

Received: 30 November 2017

Accepted: 10 August 2018

\begin{abstract}
A signed graph is a graph whose edges carry the weight ' + ' or ' - '. A signed graph $S$ is called signed-regular if $d^{-}(v)$ is same for all $v \in V$ and $d^{+}(v)$ is same for all $v \in V$. The problems of embedding $(i, j)$-signed-regular graphs in $(i, j+l)$-signed-regular graphs is one of the fascinating problems from application point of view, which is dealt in this paper with insertion of least number of vertices in $S$.
\end{abstract}

Keywords: Signed graph, Signed-regular graph, Embedding.

2010 Mathematics Subject Classification: 05C22, 05 C60.

\section{Introduction}

Embedding of the graph has regularly been studied in many areas as communications, automotive, medical, military purposes and so on. Optimal distortion embedding of distance regular graphs has been studied into Euclidean space which is extensively used to design approximation algorithms. Even in daily life it is used extensively in cellphones, digital cameras, MP3 players, portable digital assistants and automobile antilock brake systems, etc. Seeing its importance and its studies by various authors in the literature ([3], [8-17], [21]) we were motivated towards working out theoretically the embedding signed-regular graphs in higher order signed-regular graphs.

To better understand the paper, for the preliminary notation and terminology Behzad and Chartrand [4], Harary [7], West [20] and Zaslavsky [22-24] are referred. A signed graph is an ordered pair $S=\left(S^{u}, \sigma\right)$, where $S^{u}=(V, E)$ is a graph, called the underlying graph of $S$ and $\sigma: E \rightarrow\{+,-\}$ is a function from the edge set $E$ of $S^{u}$ into the set $\{+,-\} . \sigma(e)$ is then said to be the sign of $e$. The set $\left\{e \in E\left(S^{u}\right): \sigma(e)=+\right\}$ and $\left\{e \in E\left(S^{u}\right): \sigma(e)=-\right\}$ 
are called the set of positive and negative edges of $S$, respectively. If for every edge $e \in E\left(S^{u}\right)$, $\sigma(e)=+(\sigma(e)=-)$, then $S$ is said to be homogeneous, and if it is not the case, then $S$ is said to be heterogeneous.

Edges in the graph are said to be adjacent if they are incident to a common vertex and independent if no two of them are adjacent. By a matching in a graph $G$, we mean an independent set of edges in $G$. The edge independence number $\beta_{1}(G)$ of a graph $G$ is the maximum cardinality of an independent set of edges. $\overline{S^{u}}$ is said to be the complement of a graph $S^{u}$ when $E\left(S^{u}\right) \cup E\left(\overline{S^{u}}\right)=E\left(K_{n}\right)$, when $V\left(S^{u}\right)=V\left(\overline{S^{u}}\right),|V|=n$ and $E\left(S^{u}\right) \cap E\left(\overline{S^{u}}\right)=\Phi$. A graph is called $r$-regular if all its vertices are of degree $r$. A signed graph $S$ is called signed-regular if $d^{-}(v)$ is the same for all $v \in V(S)$ and $d^{+}(v)$ is the same for all $v \in V(S)$ (see [18]).

A $u-v$ path in a signed graph $S$ is an alternating sequence of vertices without repetition, beginning with $u$ and ending at $v$ such that consecutive vertices in the sequence are adjacent, and we can express it as $u=v_{0}, v_{1}, \ldots, v_{k}=v$. A path is called homogeneous if all its edges have the same sign and heterogeneous otherwise. The number of edges in a path is called the length of the path. $P_{n}$ denotes a path of length $n-1 . P_{n}^{-}\left(P_{n}^{+}\right)$denotes all the negative (positive) path of length $n-1$.

The Cartesian product $S^{u} \square S^{u}{ }_{2}$ of two graphs $S^{u}{ }_{1}$ and $S^{u}{ }_{2}$ is a graph with vertex set $V\left(S_{1}^{u}\right) \times V\left(S_{2}^{u}\right)$ and two vertices $\left(u_{1}, u_{2}\right)$ and $\left(v_{1}, v_{2}\right)$ are adjacent in $S^{u}{ }_{1} \square S^{u}{ }_{2}$ if and only if $u_{1}$ is adjacent to $v_{1}$ in $S^{u}{ }_{1}$ and $u_{2}=v_{2}$ or $u_{2}$ is adjacent to $v_{2}$ in $S_{2}{ }_{2}$ and $u_{1}=v_{1}$. Since in tensor product of graphs $S^{u}{ }_{1}$ and $S^{u}{ }_{2}$, the degree of a vertex $(u, v)$ is defined as $\operatorname{deg}(u, v)=\operatorname{deg}(u) \operatorname{deg}(v)$, if $S^{u}{ }_{1}$ and $S^{u}{ }_{2}$ are regular, then $S^{u} \square S^{u}{ }_{2}$ is also regular.

Let $S=\left(S^{u}, \sigma\right)$ be a signed graph. $S$ is called Cartesian product of two signed graphs $S_{1}=\left(S_{1}^{u}, \sigma_{1}\right)$ and $S_{2}=\left(S_{2}^{u}, \sigma_{2}\right)$ if $S^{u} \cong S_{1}^{u} \square S_{2}^{u}$ and for any edge $\left(u_{1}, u_{2}\right)\left(v_{1}, v_{2}\right)$ of $S^{u}$,

$$
\sigma\left(\left(u_{1}, u_{2}\right)\left(v_{1}, v_{2}\right)\right)= \begin{cases}\sigma_{1}\left(u_{1} v_{1}\right) & \text { if } u_{2}=v_{2}, \\ \sigma_{2}\left(u_{2} v_{2}\right) & \text { if } u_{1}=v_{1} .\end{cases}
$$

The Cartesian product of two signed graphs $S_{1}$ and $S_{2}$ is shown in Figure 1 .

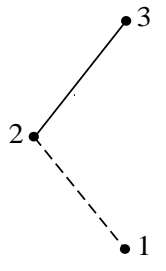

$\mathrm{S}_{1}$

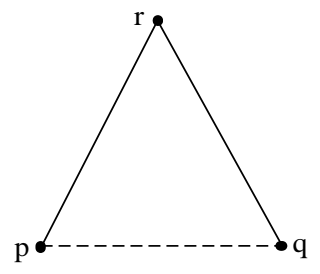

$\mathrm{S}_{2}$

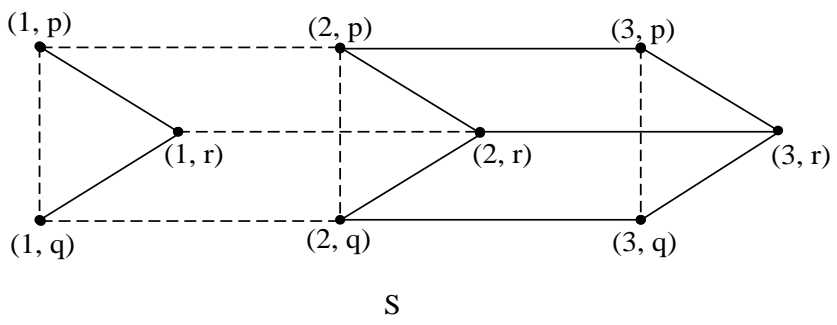

Figure 1. $S=S_{1} \square S_{2}$. 


\section{Embedding a signed regular graphs}

For a graph $G^{\prime}$, embedding of $G$ into a graph $G^{\prime}$ we mean that there exists a subgraph of $G^{\prime}$ which is isomorphic to $G$. It is generally denoted as $G \subseteq G^{\prime}$ (see [1]). Embedding, $G \subseteq G^{\prime}$ is said to be proper or strict if $G \subset G^{\prime}$. Now we embark upon the finding of the results about the embedding of signed-regular graphs. Some of the results obtained here were presented in the IEEE sponsored conference at Dhaka, Bangladesh [19].

Note: Henceforth, throughout the paper, we consider $S=\left(S^{u}, \sigma\right)$ to be an $(i, j)$-signed-regular graph of order $n$.

Gardiner (see [6]) made an observation and also gave proof to the fact that in case of graph $G$, when $G$ is $r$-regular, it can be embedded in $G \square P_{2}$ which is $(r+1)$-regular graph. Embedding of a 2-regular graph $G$ in a 3-regular graph $G=H \square P_{2}$ is shown in Figure 2. Then following can be easily observed in signed graphs.

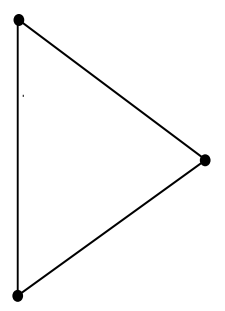

$\mathrm{H}$

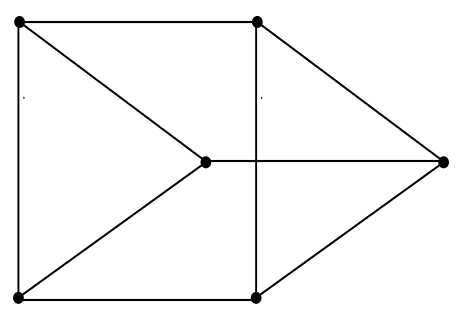

G

Figure 2. $G=H \square P_{2}$.

Remark 2.1. If a signed graph $S$ is $(i, j)$-regular, then $S \square P_{2}^{-}$is $(i, j+1)$-regular and contains $S$ as an induced sub-signed graph.

The important thing that one can think about is the optimal number of vertices to be added to $S$ to obtain such kind of embedding. Let the number of extra vertices added be denoted by $\ell(S)$.

\section{Embedding of an $(i, j)$-signed-regular graph in an $(i, j+1)$-signed-regular graph}

Theorem 3.1. For a signed graph $S$, if $\overline{S^{u}}$ has a 1-factor, then $\ell(S)=0$.

Proof. Since $\overline{S^{u}}$ has a 1-factor, add all the edges of 1-factor of $\overline{S^{u}}$ in $S$ with negative sign. Thus, we get an $(i, j+1)$-signed-regular graph without increasing the number of vertices of $S$. Hence $\ell(S)=0$.

Corollary 3.2. For $S=\left(C_{2 n}, \sigma\right)$, where $C_{n}$ denotes a cycle of order $n, \quad \ell(S)=0$.

Proof. Since $\overline{C_{2 n}}$ has a 1-factor, due to Theorem 3.1, $\ell(S)=0$. 
Corollary 3.3. For $S=\left(K_{n, n}, \sigma\right), \ell(S)=0$ if $n$ is even.

Proof. Since $\overline{K_{n, n}}$ has a 1 -factor for even values of $n$, due to Theorem $3.1, \ell(S)=0$.

Theorem 3.4. For a signed graph $S$, if $\overline{S^{u}}$ has no 1-factor, then $i+1 \leq \ell(S) \leq n$.

Proof. Since $\overline{S^{u}}$ has no 1 -factor, we have to generate positive regularity $i$ with the new added vertices. Thus,

$$
\ell(S) \geq i+1
$$

From Remark 2.1,

$$
\ell(S) \leq n
$$

Using equations (1) and (2),

$$
i+1 \leq \ell(S) \leq n
$$

Theorem 3.5. [5] For a graph $G$ of order $n$, if $\delta=\Delta$ is even, then $G$ has at least $\left\lceil\frac{n \Delta}{2(\Delta+1)}\right\rceil$ independent edges, where $\delta(G)(\Delta(G))$ denotes minimum (maximum) degree in a graph $G$.

If $K_{i+j+1}^{(i, j)}$ denotes $(i, j)$-signed-regular complete signed graphs of order $i+j+1$, then one can think of improving the bound talked about in Theorem 3.4. Theorem 3.6 below gives the solution to the same.

Theorem 3.6. [19] For a signed graph $S$ having no 1 -factor in $\overline{S^{u}}$ and $n$ and $i+j$ are of opposite parity, $\ell(S) \leq i+j+1$, where $|V(S)|=n$.

Proof. Let $i+j+1$ be the vertices required to form the $(i, j)$-signed-regular complete graph $K_{i+j+1}^{(i, j)}$. If $\overline{S^{u}}$ is a $(n-i-j-1)$-regular graph, where $(n-i-j-1)$ is even, then the number of independent edges in $\overline{S^{u}} \geq \frac{n-i-j-1}{2}$, by Theorem 3.5.

Thus, by selecting $\frac{n-i-j-1}{2}$ independent edges $e_{1}, e_{2}, \ldots, e_{\frac{n-i-j-1}{2}}$ in $\overline{S^{u}}$ and increasing the negative degree by one, inserting these independent edges in $S$ with negative sign. The remaining $i+j+1$ vertices of $S$ are joined by a negative edge to the vertices of $K_{i+j+1}^{(i, j)}$ in one-to-one manner. In a similar manner, we join all $i+j+1$ vertices of $S$ to the vertices of $K_{i+j+1}^{(i, j)}$, illustrated in Figure 3. Thus, the resulting signed graph is $(i, j+1)$-signed-regular. Hence $\ell(S) \leq i+j+1$.
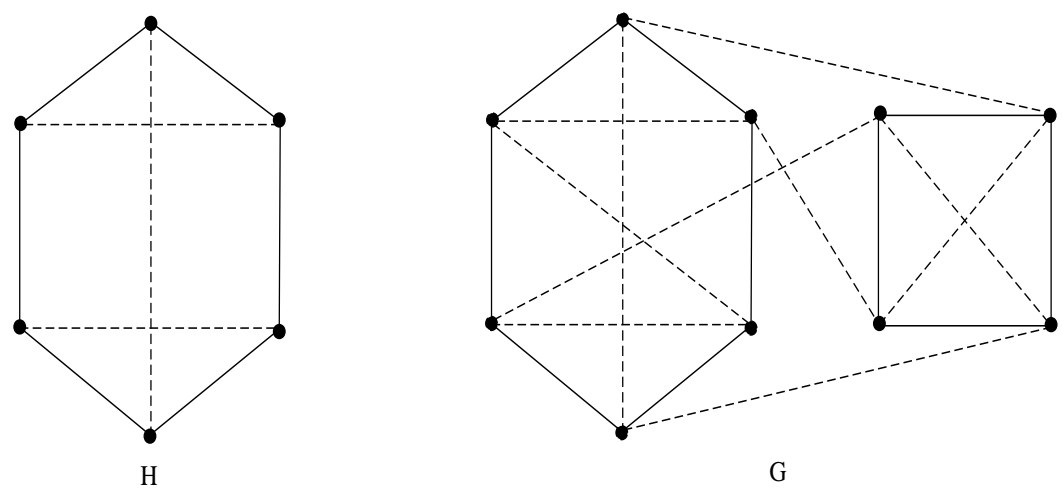

Figure 3. $H \subset G$. 
Remark 3.7. Alternatively, the proof of Theorem 3.6 can be written as:

Proof. Let $M$ be a maximum matching in $\overline{S^{u}}$, so that $|M|=\beta_{1}\left(\overline{S^{u}}\right)$ and $A$ denote the set of all $M$ saturated vertices. Then $A=2|M|$. Now let $S$ be a $(i, j)$-signed-regular graph, using the results of Theorem 3.5, the number of independent edges in $\overline{S^{u}} \geq \frac{n-i-j-1}{2}$, i.e., $\beta_{1}\left(\overline{S^{u}}\right) \geq \frac{n-i-j-1}{2}$. Let us take $\beta_{1}\left(\overline{S^{u}}\right)=\frac{n-i-j-1}{2}$. By adding the edges of $\beta_{1}\left(\overline{S^{u}}\right)$ to $S$ with a negative sign, the $2 \beta_{1}$ vertices have degree $(i, j+1)$. For the remaining $n-2 \beta_{1}$ vertices, i.e., $i+j+1$ we have to choose $K_{i+j+1}^{(i, j)},(i, j)$-regular complete signed graph. Now add a matching of $i+j+1$ negative edges joining the $i+j+1$ vertices of $S$ and a set of $i+j+1$ vertices of $K_{i+j+1}^{(i, j)}$. Thus, the resulting signed graph is $(i, j+1)$-regular. Hence, $\ell(S) \leq i+j+1$.

Theorem 3.8. For a signed graph $S$ having no 1-factor in $\overline{S^{u}}$ and $n$ and $i+j$ are of opposite parity, then $\ell(S)=i+1$, where $|V(S)|=n$ and $n \geq(i+1)(j+1)$.

Proof. By the hypothesis and Theorem 3.4, $\ell(S) \geq i+1$. In order to obtain the solution first we construct $K_{i+1}^{(i, 0)}$. Since $\overline{S^{u}}$ is a $(n-1-i-j)$-regular graph, using Theorem 3.5, the number of independent edges in

$$
\begin{aligned}
& \overline{S^{u}} \geq \frac{n-1-i-j}{2} \\
& \geq \frac{n-1-i-j-i j}{2} \\
& =\frac{n-(i+1)(j+1)}{2} .
\end{aligned}
$$

Thus, by selecting $\frac{n-(i+1)(j+1)}{2}$ independent edges $e_{1}, e_{2}, \ldots, e_{\frac{n-(i+1)(j+1)}{2}}$ in $\overline{S^{u}}$ and increase the negative degree by one by inserting these independent edges in $S$ with negative sign. Now, join by negative edges, any $j+1$ vertices among $(i+1)(j+1)$ unaffected vertices of $S$ to a vertex of $K_{i+1}^{(i, 0)}$ and join by negative edges other $j+1$ unaffected vertices of $S$ to the other vertex of $K_{i+1}^{(i, 0)}$, similarly each vertex of $K_{i+1}^{(i, 0)}$ is joined to $j+1$ vertices of $S$. The signed graph so obtained is $(i, j+1)$-regular. Hence, $\ell(S)=i+1$.

Theorem 3.9. For a signed graph $S$ having no 1 -factor in $\overline{S^{u}}$, where $n$ is even and $i+j$ is odd, such that $i \geq 1$, then

$$
\ell(S)= \begin{cases}i+j+1 & \text { if } n=i+j+1, i+j+3, \ldots, 2 i+2 j-2, \\ i+j & \text { if } n=2 i+2 j, i=2,4, \ldots, \frac{n}{2}-j \\ i+j+1 & \text { if } n=2 i+2 j, i=3,5, \ldots, \frac{n}{2}-j .\end{cases}
$$

Proof. Suppose $n \leq 2 i+2 j-2$. We shall show that $\ell(S)=i+j+1$. If possible $\ell(S) \leq i+j$. First we construct $K_{i+j}^{(i, j-1)}$. In order to obtain the solution $n$ must be at least $2(i+j)$, contradicting the assumption. This implies $\ell(S) \geq i+j+1$. Also from Theorem 3.6, $\ell(S) \leq i+j+1$. Thus, from above inequalities $\ell(S)=i+j+1$.

Next, suppose $n=2 i+2 j, i=2,4, \ldots, \frac{n}{2}-j$. If possible $\ell(S) \leq i+j-1$. Now we construct $K_{i+j-1}^{(i, j-2)}$. In order to obtain the required graph, $n$ should be at least $3(i+j-1)$, which 
contradicts the assumption except for the case $i=2, j=1$. For $i=2, j=1$, we require at least $i+1$, i.e. $i+j$ new vertices. Thus, $\ell(S) \geq i+j$.

From Theorem 3.6, $\ell(S) \leq i+j+1$.

Now we construct $K_{i+j}^{(i, j-1)}$. Join by negative edges any two vertices among $2(i+j)$ vertices of $S$ to a vertex of $K_{i+j}^{(i, j-1)}$ and join by negative edges any other two vertices of $S$ to a vertex of $K_{i+j}^{(i, j-1)}$. Hence the signed graph so obtained is $(i, j+1)$-regular. Hence,

$$
\ell(S)=i+j .
$$

Again, suppose $n=2 i+2 j, i=3,5, \ldots, \frac{n}{2}-j$. Since $i$ and $(i+j)$ both are odd, assume $\ell(S) \leq i+j-1$. Now to obtain the required solution one can construct $K_{i+j-1}^{(i, j-2)}$. Therefore, $n$ should be at least $3(i+j-1)$, which contradicts the assumption except for $i=3, j=0$. For $i=3, j=0$, we require at least $i+1$, i.e., $i+j+1$ new vertices. Thus,

$$
\ell(S) \geq i+j .
$$

From Theorem 3.6, $\ell(S) \leq i+j+1$.

Since $i$ and $(i+j)$ both are odd, therefore a $K_{i+j}^{(i, j-1)}$ regular graph is not possible. Therefore, $\ell(S)=i+j$ is not possible.

This implies,

$$
\ell(S)=i+j+1
$$

The Theorem 3.9 is illustrated in Figure 4.

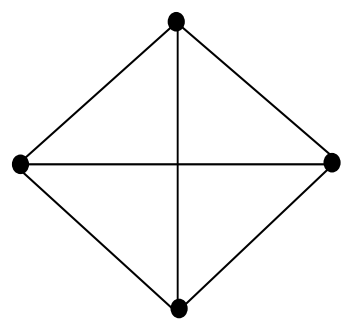

$\mathrm{H}$

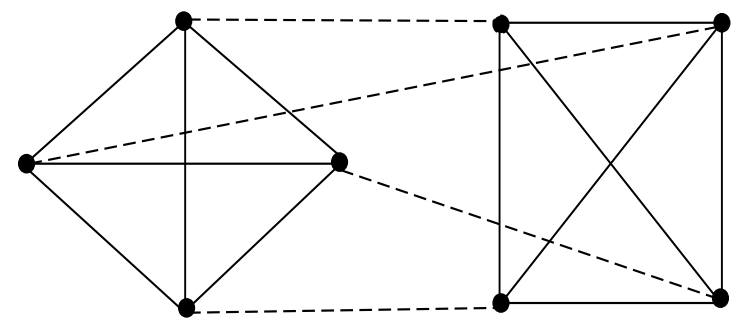

G

Figure 4. $H \subset G$.

Proposition 3.10. For a signed graph $S=\left(K_{n, n}, \sigma\right)$, if $n$ is odd, then

$$
\ell(S)= \begin{cases}2 & \text { if } i=1, \\ i+j & \text { if } i=2,4, \ldots, i+j-1 \\ i+j+1 & \text { if } i=3,5, \ldots, i+j-2 .\end{cases}
$$

Theorem 3.11. [20] If $G$ is a simple connected graph of order $n$ with $n \geq 3$ and $d(v) \geq \frac{n}{2}$ $\forall v \in V(G)$, then $G$ is a Hamiltonian graph.

Theorem 3.12. For a signed graph $S$ if $n \geq 2 i+2 j+2$ is even, then $\ell(S)=0$. 
Proof. Since $\overline{S^{u}}$ is $(n-1-i-j)$-regular graph of order $n$,

$$
\begin{gathered}
d(v)=n-(1+i+j) \forall v \in V\left(\overline{S^{u}}\right) \\
\geq n-\frac{n}{2} \forall v \in V\left(\overline{S^{u}}\right) \\
=\frac{n}{2} \forall v \in V\left(\overline{S^{u}}\right) .
\end{gathered}
$$

Thus, $d(v) \geq \frac{n}{2} \forall v \in V\left(\overline{S^{u}}\right)$. Due to Theorem 3.11, $\overline{S^{u}}$ is a Hamiltonian graph. Since the order of $\overline{S^{u}}$ is even and it is Hamiltonian, $\overline{S^{u}}$ has a 1-factor. Now due to Theorem 3.1, $\ell(S)=0$.

Theorem 3.13. [6] Let $G$ be a $r$-regular graph on $n$ vertices. If $\bar{G}$ has no 1-factor and $n, r$ have the same parity, then $n<2 r$ and $\ell(S)=r+2$.

Theorem 3.14. For a signed graph $S$ having no 1 -factor in $\overline{S^{u}}$, where $n$ is odd and $i+j$ is even and $i \geq 1$, then

$$
\ell(S)= \begin{cases}i+j+1 & \text { if } n<3(i+j-1), \\ i+j-(r-2) & \text { if } n=r(i+j-k), k \text { and } r \text { are odd } .\end{cases}
$$

Proof. When $i$ and $j$ both are odd, then the graph is not possible since $n$ is odd. This implies $i$ and $j$ both are even.

Suppose $n<3(i+j-1)$, we will show that $\ell(S)=i+j+1$. If possible $\ell(S)<i+j$. Now we construct $K_{i+j}^{(i, j-1)}$. The least value $n$ can take is $2(i+j)$ to obtain an $(i, j+1)$-signed-regular graph; for if $n<2(i+j)$, then we have a contradiction to the assumption. Hence,

$$
\ell(S) \geq i+j+1 \text {. }
$$

If $2(i+j)<n<3(i+j-1)$ and $\ell \leq i+j-1 . K_{i+j-1}^{(i, j-2)}$ is constructed. The least $n$ possible is $3(i+j-1)$ for the signed graph to be an $(i, j+1)$-signed-regular, which is again a contradiction to the assumption. Hence,

$$
\ell(S) \geq i+j .
$$

Since the least number of independent edges in $\overline{S^{u}} \geq \frac{n(n-i-j-1)}{2(n-i-j)}>\frac{n-i-j-1}{2}>\frac{n-2(i+j)-1}{2}$. Thus by selecting $\frac{n-2(i+j)-1}{2}$ independent edges $e_{1}, e_{2}, \ldots, e_{\frac{n-2(i+j)-1}{2}}$ in $\overline{S^{u}}$ and increase the negative degree by one by inserting these independent edges in $S$ with a negative sign. Now from the remaining $2(i+j)+1$ vertices after joining by negative edge $i+j$ vertices of $K_{i+j}^{(i, j-1)}$ to $2(i+j)$ vertices of $S$ we are left with one vertex $v$ (say) such that $d^{-}(v)=j$. Hence,

$$
\ell(S) \geq i+j+1
$$

From Theorem 3.6, equation (4) and (3), we get $\ell(S)=i+j+1$.

Suppose $n \geq r(i+j-k), j \geq r-1 ; k=1,3, \ldots, r=k+2, k+4, \ldots$. Now construct $K_{i+j-(r-2)}^{(i, j-(r-3))}$.

The number of the independent edges in

$$
\overline{S^{u}} \geq \frac{n(n-(i+j+1))}{2(n-(i+j))} \geq \frac{n-(i+j+1)}{2} \geq \frac{n-(r-2)(i+j-(r-2))}{2},
$$


and $r>3$.

If $r=k+2$, then we construct $K_{i+j-k}^{(i, j-(k+1))}$ and $n=(k+2)(i+j-k)$. Thus to obtain an $(i, j+1)$-signed-regular graph one can join by a negative edge $(k+2)$ vertices of $S$ to a vertex of $K_{i+j-k}^{(i, j-(k+1))}$ and join by negative edge other $(k+2)$ vertices of $S$ to the other vertex of $K_{i+j-k}^{(i, j-(k+1))}$ with a negative edge. The signed graph so obtained is an $(i, j+1)$-signed-regular. Hence, $\ell(S)=i+1$.

If $r=k+4, k+6, \ldots$. Thus by selecting $\frac{n-(r-2)(i+j-(r-2))}{2}, r>3$ independent edges $e_{1}, e_{2} \ldots e_{\frac{n-(r-2)(i+j-(r-2))}{2}}$ in $\overline{S^{u}}$ and increasing the negative degree by one by inserting these independent edges in $S$ with a negative sign.

Now join by negative edges remaining $(r-2)(i+j-(r-2))$ vertices of $S$ to the vertices of $K_{i+j-(r-2)}^{(i, j-(r-3))}$ by joining $(r-2)$ vertices to a vertex of $K_{i+j-(r-2)}^{(i, j-(r-3))}$ and join by negative edges other $(r-2)$ unaffected vertices of $S$ to another vertex of $K_{i+j-(r-2)}^{(i, j-(r-3))}$. Thus the signed graph so obtained is an $(i, j+1)$-signed-regular graph. Hence,

$$
\ell(S)=i+j-(r-2) .
$$

Theorem 3.15. For a signed graph $S$ having no 1-factor in $\overline{S^{u}}$, where $n$ and $i+j$ are of same parity, then $\frac{n}{2}<(i+j) \leq n-4$ and $\ell(S)=i+j+2$.

Proof. It is obvious that both $n$ and $i+j$ should be even. Suppose $\ell(S) \leq i+j+1$. Now one can construct $K_{i+j}^{(i, j-1)}$. The least $n$ possible is $2(i+j)$ for the signed graph to be $(i, j+1)$-signedregular, which is again a contradiction to the assumption. Now we construct $K_{i+j+1}^{(i, j)}$, and join by negative edge $i+j+1$ vertices of $K_{i+j+1}^{(i, j)}$ to $i+j+1$ vertices of $S$. Then we are left with one vertex of negative degree $j$, which implies

$$
\ell(S) \geq i+j+2 .
$$

Due to Theorem 3.5, the number of independent edges in $\overline{S^{u}} \geq \frac{n-i-j-1}{2} \geq \frac{n-i-j-2}{2}$.

Thus by selecting $\frac{n-i-j-2}{2}$ independent edges $e_{1}, e_{2}, \ldots, e_{\frac{n-i-j-2}{2}}$ from $\overline{S^{u}}$ and increasing the negative degree by one by inserting these independent edges in $S$ and join by negative edge remaining $i+j+2$ vertices of $S$ to vertices of an $(i, j)$-signed-regular graph of $i+j+2$ vertices. Therefore,

$$
\ell(S)=i+j+2 .
$$

Theorem 3.16. For a signed graph $S=\left(S^{u}, \sigma\right)$ where both $n$ and $i+j$ are of same parity, if $\frac{n}{2} \geq(i+j)$ or $(i+j)=n-2$, then $\ell(S)=0$.

Proof. If $n=2(i+j)$, then $\overline{S^{u}}$ is $(i+j-1)$-regular. If $\overline{S^{u}}$ is 2-connected then $\overline{S^{u}}$ is Hamiltonian. While if $\overline{S^{u}}$ is not 2-regular, then $\overline{S^{u}} \cong 2 K_{i+j}$. In either case $\overline{S^{u}}$ has 1 -factor and due to Theorem $3.1, \ell(S)=0$.

If $n \geq 2(i+j+1)$, then $d\left(u_{i}\right) \geq 2(i+j+1)-(i+j+1) \geq \frac{n}{2}$, for every $u_{i} \in \overline{S^{u}}$.

Therefore $\overline{S^{u}}$ is Hamiltonian. This implies $\ell(S)=0$.

When $n=i+j+2$, then degree of every vertex in $\overline{S^{u}}$ is one, which implies $\overline{S^{u}}$ has 1-factor and hence, $\ell(S)=0$.

The Theorem 3.16 is illustrated in Figure 5. 


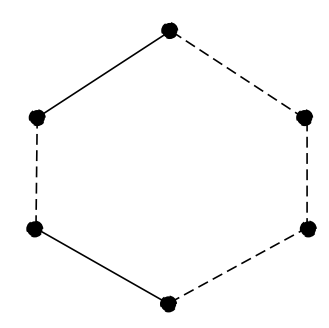

$\mathrm{H}$

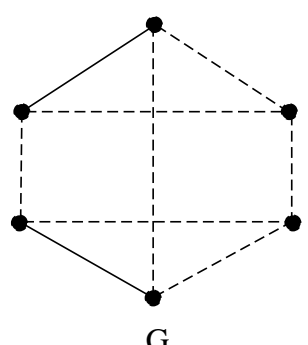

G

Figure 5. $H \subset G$.

\section{Conclusion}

The proof of all the Theorems in this paper can also be worked out by an alternative method of matching as we have shown it in the proof of Theorem 3.6. It is a well-posed problem in graph theory in the realm of embedding that every graph can be embedded as a subgraph in a regular graph and also it remains induced. Late B. D. Acharya in personal communication in 2012 [2] inspired the authors to work on the following conjecture:

Conjecture-1 (B. D. Acharya): Every signed graph with maximum positive degree $\Delta^{+}$and maximum negative degree $\Delta^{-}$can be embedded in a $\left(\Delta^{+}+1, \Delta^{-}+1\right)$-signed-regular graph.

Results which deal with the proof of the above mentioned Conjecture- 1 for $(i, j)$-signed-regular graph are dealt elsewhere.

\section{Acknowledgements}

The authors are very much thankful to the late Dr. B. D. Acharya for motivating them to work on more results on this idea of embedding of signed graphs with different aspect other than regularity. This research is supported by University grants commission (Govt. of India), New Delhi, India (21/06/2015(i)EU-V).

\section{References}

[1] Acharya, B. D. (1982) Construction of certain infinite families of graceful graphs from a given graceful graph, Def. Sci. J., 32(3), 231-236.

[2] Acharya, B.D. Personal communication, 2012.

[3] Archdeacon, D. (1990) The complexity of the graph embedding problem, in: Topics in Combinatorics and Graph Theory, R. Bodendiek and R. Henn (Eds.), Physica-Verlag, Heidelberg, 1990, 59-64.

[4] Behzad, M., \& Chartrand, G. (1971) Introduction to the Theory of Graphs, Allyn and Bacon, Inc., Boston. 
[5] Bollobas, B., \& Eldridge, S. E. (1976) Maximal matchings in graphs with given minimal and maximal degrees, Math. Proc. Cambridge Philos. Soc., 79, 221-234.

[6] Gardiner, A. (1983) Embedding $k$-regular graphs in $k+1$-regular graphs, J. London Math. Soc., 2, 28, 393-400.

[7] Harary, F. (1969) Graph Theory, Addison-Wesley Publ. Comp., Reading, Massachusetts.

[8] Kawarabayashi, K., Mohar, B., \& Reed, B. (2008) A simpler linear time algorithm for embedding graphs into an arbitrary surface and the genus of graphs of bounded tree-width, FOCS, IEEE 49th Annual IEEE symposium, 771-780.

[9] Juvan, M., \& Mohar, B. (1997) A linear time algorithm for the 2-M obius band embedding extension problem, SIAM J. Discrete Math., 10(1), 57-72.

[10] Gupta, A. K., Nelson, D., \& Wang, H. (2003) Efficient embeddings of ternary trees into hypercubes, J Parallel Distrib Comput, 63, 619-629.

[11] Manuel, P., Arockiaraj, M., Rajasingh, I., \& Rajan, B. (2011) Embedding hypercubes into cylinders, snakes and caterpillars for minimizing wire length, Discrete Appl. Math., 159, 2109-2116.

[12] Mohar, B. (1996) Embedding graphs in an arbitrary surface in linear time, Proceedings of 28th Ann. ACM STOC, Philadelphia, 392-397.

[13] Mohar, B. (1999) A linear time algorithm for embedding of graphs in an arbitrary surface, SIAM J. Discrete Math., 12, 6-26.

[14] Mohar, B. (1994) Obstructions for the disk and the cylinder embedding extension problems, Comb. Probab. Comput., 3, 375-406.

[15] Rajasingh, I., Manuel, P., Arockiaraj, M., \& Rajan, B. (2013) Embeddings of circulant networks, J Comb Optim, 26, 135-151.

[16] Raman, I., \& Choudum, S.A. (2013) Embedding certain height-balanced trees and complete $p^{m}$-ary trees into hypercubes, $J D A, 22,53-65$.

[17] Singh, T., \& Acharya, M. (2013) Embedding of sigraphs in graceful sigraphs, ARS Combinatoria, 111, 421-426.

[18] Sinha, D., \& Garg, P. (2011) On the regularity of some signed graph structures, $A K C E$ International Journal of Graph and Combinatorics, 8, 1, 63-74.

[19] Sinha, D., Rao, A. K., \& Garg, P. (2016) Embedding of $(i, j)$-Regular Signed Graphs in $(i+k, j+l)$-Regular Signed Graphs, Proceedings of IEEE explore, 2016 International Workshop on Computational Intelligence, Dhaka, Bangladesh, 12-13 December 2016, 10.1109/IWCI.2016.7860368. 
[20] West, D. B. (1996) Introduction to Graph Theory, Prentice-Hall of India Pvt. Ltd., India.

[21] Zaslavsky, T. (1992) Orientation embedding of signed graphs, J. Graph Theory, 16, 5, 399422.

[22] Zaslavsky, T. (1982) Signed graphs, Discrete Math., 4, 47-74.

[23] Zaslavsky, T. (1998) Glossary of signed and gain graphs and allied areas, Electron. J. Combin., II Edition, 1083-1224.

[24] Zaslavsky, T. (1998) A mathematical bibliography of signed and gain graphs and allied areas, Electron. J. Combin, VII Edition, 157 pp. 